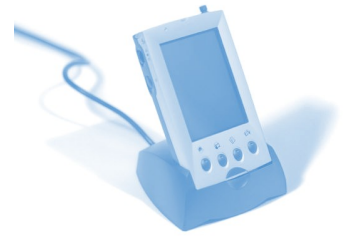

David Sands* and

Tina Overton Department of Physical

\section{Sciences}

University of Hull

Hull

HU6 7RX

*d.sands@hull.ac.uk

\title{
Cognitive psychology and problem solving in the physical sciences
}

\begin{abstract}
This paper provides and introduction to the literature on cognitive psychology and problem solving in physical sciences. We consider the working memory and its three different components, two of which hold and record information and are controlled by an executive that controls attention. Working memory alone cannot explain problem solving ability and we review the influence of schemata, the construction of mental models, visual reasoning and the cognitive style of field dependence.
\end{abstract}

\section{Introduction}

The ability to solve problems is widely recognised as an important outcome of undergraduate programmes. For example, in 2008 alone there were over 160 papers published in over 100 different journals which covered topics as diverse as child development, psychology, cognition, computers, neurosciences, mathematics and education ${ }^{1}$. The breadth is quite staggering, but we are interested mainly in problems in chemistry and physics. Intuitively we feel that problem solving in these areas is likely to be different from problem solving in, say, mathematics or medicine. Is it, though? Or is it just that the content is different but the techniques are similar? Specifying exactly what it is we do when solving problems is difficult enough, but defining exactly what we should be teaching and how seems nigh on impossible.

The first question that inevitably arises is, what do we mean by problem solving? Hayes wrote in $1980^{2}:$ ' Whenever there is a gap between where you are now and where you want to be, and you don't know how to find a way to cross that gap, you have a problem.' A similar view was expressed by Wheatley': 'Problem solving is what you do when you don't know what to do.' Pithy, but seemingly not too helpful. However, they express the essential difficulty: if you're at a loss, how do you get started? Perhaps specific help can be offered in specific circumstances, but it would be better if we could identify the essential elements of a problem solving strategy. As we will show, knowledge is important, but knowledge alone is not enough. Can we identify what else is needed and change the way we teach to cultivate problem solving abilities? This paper will attempt to answer these questions by drawing on the literature from cognitive psychology and both physics and chemistry education research to lay down some general principles of problem solving.

\section{Expert-Novice studies}

Much of the early literature on problem solving were concerned with general processes and rules for reasoning and problem solving '... that might be acquired as transferable habits of thinking' ${ }^{4}$. The content of such studies were abstract problems, or perhaps puzzles, that had little direct connection with the domains of knowledge with which real-life problem solvers were familiar and in which they operated. It was not until the late 1970s that the artificial intelligence (AI) community began to construct computer programmes of problem solving and the importance of domain-specific knowledge, and in particular the organising principles behind the knowledge structures of experts, was recognised. Physics was identified as an established problem-solving discipline and provided the context in which many of the early expert-novice studies were undertaken.

Two papers stand out from that time by Larkin et $a l^{5}$ and by Chi et $a f^{6}$. Larkin et al were concerned to produce computer programmes that replicated human thinking and described a few examples, at the heart of which are production rules. These are specific algorithms that generate actions from given conditions: 'if $x$ then $y$ '. This paper is remarkable for its early recognition of all the important features of problem solving that have dominated the literature since, including in particular working memory capacity and the use of representations, both internal and external. Working memory capacity is essentially how much information can be held in the head at any one time. Larkin et al 
described expert chess players who can memorise with remarkable accuracy the positions of some 25 or so chess pieces on a board after looking at them for only a few seconds. Novices, on the other hand, can remember only a few pieces, but experts perform in much the same way as novices if the pieces are placed randomly. Experts are familiar with many different patterns of play and clearly recognise and recall those patterns, thereby reducing the load on working memory. Experts in physics employ similar strategies, working forward from the givens to the solutions on simple kinematics problems. Novices, or students, tend to work backwards and according to Larkin et al this is a more efficient search strategy. Clearly experts are not searching; they are recognising patterns and working with them.

Chi et al discuss many of the same things, but they were concerned to look also at how knowledge is structured. Rather than solving problems, they asked experts and novices to characterise a set of end-of-chapter problems according to common features. Experts characterised the problems according to principles or laws involved in their solution, whereas students characterised them according to common surface features. In Glaser's words ${ }^{4}$; “... [the] knowledge of novices is organised around the literal objects explicitly given in a problem statement. Experts' knowledge, on the other hand, is organized around principles and abstractions that subsume these objects." Access and use of knowledge is "a major component of thinking" according to Glaser", but there is one aspect of thinking that is not really addressed in any of these papers: that is the use of qualitative reasoning. Much is made of content knowledge: concepts, laws, facts, etc. and the procedural and strategic

knowledge of how, where and when to use it. But the problems in these studies are quantitative, so the methods used to solve the problem are also quantitative. However, according to di Sessa", "qualitative controls quantitative". In other words, it is much easier to arrive at a quantitative solution if first there is a qualitative understanding. Equations might symbolise concepts or laws but those symbols must be connected to "richly elaborated mental constructs" which are qualitative in nature. It is these qualitative relationships that allow us to plan and execute a problem-solving strategy.

\section{Representations}

A representation of a problem is an essential component of the mental constructs to which di Sessa refers. Experts spend a great deal more time than do novices on understanding the problem, and an essential part of that understanding is drawing a diagram. Glaser ${ }^{4}$ calls a representation constructed in order to understand a problem a 'cognitive structure'. That is to say, the construction of a representation is part of our thinking about a problem, from understanding through to the formulation of a solution. There are two kinds of representation of particular interest: diagrams and mental images. In Nersessian's view ${ }^{8}$, these two constitute a coupled system. That is to say, we use diagrams and mental images as the basis of a form of qualitative reasoning whish Nersessian calls 'model-based reasoning'.

It comes as something of a surprise to many science educators to find that diagrammatic representation and reasoning has been a topic of study for many years within the Al community. There is too much work to quote here, so we will restrict ourselves to a few illustrative examples. Of particular relevance is the work of Suwa and Tversky ${ }^{9}$, who, studying the way that designers use diagrams, concluded that diagrams contribute to the dynamic construction of ideas. In particular, diagrams free working memory by putting down on paper ideas and concepts that might otherwise have to be held in the head. Diagrams also cue retrieval from long term memory through the recognition of particular structures or patterns, allow perceptual judgements about spatial relations, and, most perhaps most important of all, allow the generation of new ideas.
Within the physical sciences education research literature there is also extensive evidence for the use of diagrams and mental representations both to understand a problem and to formulate a solution. One of the criticisms often levelled at early expert-novice studies is that the problems do not represent much of a challenge to experts who, by and large, are familiar with the material. Singh ${ }^{10}$ conducted a study in which he presented experts in physics with a problem to which they did not know the solution. He observed that diagrams were often constructed, but in addition experts use other techniques to develop an answer, including consideration of limiting cases. In order to do this it is

necessary to create and run a mental model. This is Nersessian's model-based reasoning. Within Chemistry Bodner's work stands out ${ }^{11}$, as does the work of Stieff and Raje $^{12}$. The latter presented experts with problems in organic chemistry from finals papers from a range of universities. The aim of their paper was ostensibly to show that experts use techniques other than visualisation. The technique used most was recall of either the problem or the solution, which of course obviates the need to visualise or draw a diagram. The next most common techniques were either visualisation or the use of a diagram.

\section{Spatial ability: the role of working memory}

The ability to manipulate mental images in the manner just described varies from person to person and is closely related to working memory ${ }^{13}$. Working memory capacity has already been mentioned, and the concept is fairly well known within science education, but capacity is only one aspect. According to Baddely ${ }^{14}$, the originator of the concept, the term is taken 'to apply to a limited capacity system that is capable of storing and manipulating information and that is assumed to be an integral part of the human memory system'. The concept of working memory is an extension of the earlier psychological 
concept of short term memory which was seen essentially as a temporary repository of information before it entered long term memory. Working memory, on the other hand, is not a unitary system and also has a 'functional role in other cognitive tasks such as learning reasoning and comprehension ${ }^{, 14}$.

Understanding exactly what the different components of working memory do and how they relate to each other is still an ongoing question for psychologists. It is even more difficult for non-psychologists interested in how it affects performance in science learning to understand the concept. Essentially working memory is seen as a tripartite system: two slave functions which hold and record information controlled by an executive that controls attention, how it may be switched from one focus to another, the activation of long term memory, and possibly also the processing of information. The slave functions are quite distinct, with one, the phonological loop, holding acoustic or speech-based information and the other, the visuo-spatial sketch pad, holding spatial and visual information. Despite being perceived visually, written words are still controlled by the phonological loop.

The phonological loop is the best understood component of working memory, but for our purposes it is the least interesting. In Baddeley's view ${ }^{14}$, this system probably evolved as a crucial component of language acquisition, so, beyond determining memory capacity, it is not directly involved in problem solving. Memory capacity, important as it is, is only one aspect of working memory. It is measured by speech-based information so it is connected to the phonological loop, but it is commonly supposed that there is a similar capacity for spatial or visual information. However, the visuo-spatial sketch pad is not nearly as well understood. For example, the capacity for speech-based information arises because the memory refreshes itself automatically. If refreshing is prevented, say by a distraction, the information is lost after even just a couple of seconds. In a long sequence of data, therefore, the last information is processed after the first information has disappeared from memory. All this is well known and well understood within psychology, but the visuo-spatial sketch pad is much more complex and its characteristics correspondingly harder to measure. In addition, some of its functions, especially those related to imagery, rely heavily on the central executive, which is understood even less.

Psychologists investigate the relationship between these different components through interference tests. That is, propositional reasoning is assumed to occur through the phonological loop, so if a subject is made to undertake a simple spatial secondary task it is possible to see to what extent the spatial task interferes with the primary ${ }^{15}$. If there is no interference the systems are assumed to be separate. The picture that emerges from the literature is that propositional reasoning often involves a spatial component. Complex propositions might, for example, invoke imagistic strategies such as the mental construction of Venn diagrams. It is also clear that spatial reasoning relies heavily on the central executive and that a limited capacity in the central executive can affect the ability to reason spatially and propositionally ${ }^{16,17}$. This is the basis for individual differences in spatial ability, but the implications for problem solving in the physical sciences will not be clear until the concept of field dependence-independence (FDI) has been described.

\section{Field dependence-independence}

FDI was initially used to describe the ability to perceive upright in a darkened room where the only visible objects, an illuminated square frame and a rod, may be oriented independently at an arbitrary angle ${ }^{18}$. Some subjects perceive upright to correspond to the orientation of the frame while others are able to define upright correctly regardless of what they see. People who take their cue from the visual field are said to be field dependent. It quickly emerged that field dependence defined in this way correlated with a range of other characteristics, most notably the inability to disembed important information from a perceptual field and the inability to order information. The consequences can be quite profound. First, in relation to disembedding, FD subjects often struggle to decide what is important or not in a problem statement. Second, as described by Witkin, learned material often lacks an inherent structure and, whereas field independent students are able to re-order material to aid their own learning, field dependent students struggle. Not surprisingly, a study of Spanish school children revealed a significant achievement gap across all subjects which is not accounted for by differences in general intelligence ${ }^{19}$. There are other studies that show directly, and quite emphatically, how, for field dependent students, learning in science can depend on the way material is presented and structured ${ }^{20-22}$.

These two effects are related to the central executive. Describing the role of working memory in the construction of mental models, García-Madruga states that one of the functions of the central executive is to discard irrelevant information ${ }^{16}$. Ordering information clearly places a high load on the working memory. First, several concepts have to be held in the mind at once, but in addition there has to be a fair amount of reasoning about the concepts which is likely to be more demanding than simple propositional reasoning.

Concepts have be understood not only in themselves, but also in their relationship to each other. There might even be a spatial element in as much as the concepts of before or after, first or last, following, etc. might well imply spatial relations and invoke spatial imagery. The central executive is likely to be heavily loaded during such a task and individuals with a low capacity will struggle. Rittschof ${ }^{23}$ summarises much of the psychological research which links field dependence to the central executive.

\section{Problem solving: how can it be taught?}

The preceding has laid down some of the essential ideas in cognitive psychology that relate to problem solving in the sciences. Necessarily the ideas are brief and the reader is referred to the literature for a deeper understanding. What is clear, though, is that problem solving is a complex activity and the idea that we might teach problem solving as a technique in the same way that we might teach other skills like computing does not stand up. Rather, it appears to be something that ought to be cultivated over a long time. The first requirement is for students to build up a body of knowledge, but often we want to set problems as a way of developing knowledge. This is a contradiction and probably explains why many problems are algorithmic in nature. Students simply do not have the knowledge, not to mention other skills, to cope with more complex, open-ended problems. Moreover, in trying to build up this knowledge, we run into the problem of field dependence. 
The construct originally described by Witkin is likely to be binary; either one can perceive upright independently of the visual field or one can not. However, field dependence today is often measured using a spatial reasoning test involving hidden figures and a broad spectrum of abilities is often measured, including field neutrality. The terms field dependence and independence should therefore be interpreted as tendencies rather than absolutes. That said, we have no real idea of the distribution of these tendencies among physical science students in UK universities. It is a common experience, however, that abilities vary greatly and perhaps more so now that higher education is more inclusive. If we accept that some of these students tend to field dependence we must recognise the possibility that conventional lectures will leave many of them floundering. Field dependent students appear to be very inefficient at making their own notes ${ }^{24}$. As they can neither discard irrelevant information nor impose order on perceived material they produce notes that are wordy, lack coherence, and appear to be hard to learn from. However, if they are given an outline which provides an external structure they can learn just as well as field independent

students whose notes, by contrast, tend to be in outline form, with headings, subheadings and a great deal of order to the information.

The body of knowledge should, if possible, include schemata. A schema may be considered to be a mental model around which information is organised. For example, the concept of an elastic collision implies hard spheres as well as the conservation of both momentum and energy. Thus, a schema helps to reduce the load on the working memory by allowing access to a series of associations held in long term memory. Schema are best developed by students themselves working cooperatively in a constructivist environment such as the modelling approach advocated by Hestenes ${ }^{25}$. Modelling is one form of problem solving. It often employs the sort of qualitative reasoning that we have argued is an essential component of scientific thought. As we have argued, such reasoning is not just propositional but may be spatial or diagrammatic, which brings us nicely to the subject of spatial abilities and whether they can be improved.

Spatial ability is very closely linked to field dependence and to the central executive in working memory. Spatial abilities therefore vary among individuals and ultimately might be limited by biology, but even if the capacity in the central executive or the visuo-spatial sketch pad is fixed there might be reason to suppose that strategies can be developed to compensate. Baddeley ${ }^{14}$, for example, describes patients with severe impairments to some of the functions of working memory who nonetheless cope well. The use of diagrams is likely to form part of that strategy. We have described how diagrams are used to understand problems and also to develop solutions, but crucially they reduce the load on working memory. Depending on the nature of the problem and on the individual, iconic mental models might be developed and manipulated, but if the mental models become too complicated it is necessary to free up working memory by setting some of the information down on paper. Once on paper spatial relations might be more easily perceived and investigated through the medium of the diagram.

Diagrams are important for another, perhaps unexpected, reason. Sorby ${ }^{26}$ reports that one of the best ways of developing spatial abilities is old-fashioned sketching. Perception appears to be very important for developing spatial skills. Holding and manipulating physical models, for example rotating them, also appears to work. Although these methods have been investigated in the context of engineering education, and in fact there is interest around the world ${ }^{27,28}$ in improving spatial abilities in engineering students, this is a technique that is immediately applicable to organic and molecular chemistry. It might be thought that working with 3-D computer graphics will help, but in fact it does not. It seems that there has to be some spatial reasoning associated with the task: imagery, mental rotation, mental extrapolation, etc. This is why sketching is so useful: it requires spatial reasoning to identify relationship as well as important components, which is especially interesting in light of the work by Mohler ${ }^{29}$. Mohler investigated practical differences between high and low spatial ability students by analysing their performance in a spatial task involving complicated diagrams. Even high spatial ability students can experience cognitive overload; '...I screw stuff up because when you're looking at everything, ... it's easy to get real messed up'. The difference is that students with high spatial ability recognise it and work on smaller portions of the diagram whereas those with low spatial ability do not. Mohler asks whether this skill can be taught, and as yet there is no answer. Perhaps it is a manifestation of the central executive's inability to discriminate and discard irrelevant information, but if it can be taught, perhaps sketching might be one way. In constructing a sketch it is necessary to make a choice about what is important and what is not in order to put something down on paper. Activities that encourage the construction of diagrams in problem solving do more than simply provide a mechanism for reasoning about the problem.

\section{Conclusion}

The present work has considered the cognitive psychology of problem solving from early expert-novice studies through to later studies on spatial reasoning and the cognitive style of field dependence. We have shown that qualitative reasoning about problems precedes quantitative reasoning and is an essential aspect of scientific inquiry. We have also discussed at length spatial abilities and their role in problem solving, 
particularly in constructing mental models and diagrams. It is clear that students of low spatial ability are to some extent disadvantaged in a conventional academic environment, but designing activities that will allow field independent students to flourish while at the same time providing the kind of support that will benefit field dependent students is by no means easy. According to Rittshof, "studies from several decades past emphasise the ways that field dependent learners benefit from external types of information structuring and motivation, while field independent learners benefit from the opportunity to use their own structuring of information and motivation as they construct knowledge". Serving both sets of students at the same time poses a tremendous challenge, one that perhaps has only been appreciated within the field of cognitive psychology. However, by looking at that literature as well as that from education research we can at last begin to appreciate what we should be aiming for. We have suggested how spatial skills might be cultivated, but there is a lack of practical knowledge about the best way forward.

\section{References}

1. Pizlo, Zygmunt (2008), Human Problem Solving in 2008, The Journal of Problem Solving, 2 (1), article 5.

2. Hayes, J. (1980), The complete problem solver

3. Wheatley, G H. (1984), Problem solving in school mathematics, MEPS Technical Report, School of Mathematics and Science Centre, Purdue University.

4. Glaser, Robert (1984), Education and Thinking: the role of knowledge, American Psychologist, 39 (2), 93-104.

5. Larkin, Jill; McDermott, John; Simon, Dorothea P.; Simon, Herbert A. (1980), Expert and Novice Performance in Solving physics Problems, Science, 208, 1335-1342.

6. Chi, Micheline T. H.; Feltovich, Paul J.; Glaser, Robert (1981), Categorization and Representation of Physics Problems by Experts and Novices, Cognitive Science, 5, 121-152.

7. di Sessa, Andrea (1987): The third Revolution in Computers and Education, J. Res. Sci. Teach., 24 (4), 343-367.

8. Nersessian, Nancy J. (2008), Creating Scientific Concepts, Bradford Books

9. Suwa, M. and Tversky, B. (2002), External representations contribute to the dynamic construction of ideas in M. Hegarty, B. Meyer, H. Narayanan, Diagrammatic Representation and Inference, Proc. Inference 2002, Lecture Notes in Artificial Intelligence series, Springer, 341-343.

10. Singh, C. (2002), When physical intuition fails, Am. J. Phys., 70 (11), 1103-1109.

11. Bodner, George M. and Domin, Daniel S. (2000), Mental Models: The role of representations in problem solving in chemistry, University Chemistry Education, 4 (1), 24-30

12. Stieff, M. and Raje, Sonali (2008), Expertise \& Spatial Reasoning in Advanced Scientific Problem Solving, Proc $8^{\text {th }}$ International conference on the Learning Sciences, Utrecht, NL, 2008, 366-373.

13. Rittschof, Kent A. (2008), Field dependence-independence as visuospatial and executive functioning in working memory: implications for instructional systems design and Research, Education Tech Research Dev. DOI 10.1007/s11423-008-9093-6

14. Baddeley, Alan (1996), The fractionation of working memory, Proc. Natl. Acac. Sci. USA, 93, 13468-13472.
15. Klauer, Karl Christoph; Stegmaier, Ralph; Meiser Thorsten (1997), Working memory involvement in propositional and spatial reasoning, Thinking and Reasoning, 3 (1), 9-47

16. García-Madruga, Juan A.; Gutiérrez, Francisco; Carriedo, Nuria; Luzón, José M.; Vila, José O. (2007), Mental models in propositional reasoning and working memory's central executive, Thinking And Reasoning, 13 (4), 370-393

17. Capon, Alison; Handley, Simon; and Dennis, Ian (2003), Working memory and reasoning: An individual differences perspective, Thinking And Reasoning, 9 (3), 203-244

18. Witkin, H. A.; Moore, C. A.; Goodenough, D. R.; Cox, P. W. (1977), Field-Dependent and Field-Independent Cognitive Styles and Their Educational Implications Review of Educational Research, 47 (1), 1-64.

19. Tinajero, Carolina and Páramo, M Fernanda, (1997), Field dependence-independence and academic achievement; a re-examination of their relationship, British Journal of Educational Psychology, 67, 199-212.

20. Strawitz, Barbara M. (1984): Cognitive Style and the acquisition and transfer of the ability to control variables, J. Res. Sci. Teach., 21 (2), 133-141.

21. Strawitz, Barbara M. (1984): Cognitive Style and the effects of two instructional treatments on the acquisition and transfer of the ability to control variables: a longitudinal study, J. Res. Sci. Teach., 21 (8), 833-841.

22. Antonietta, Allessandro and Gioletta, Maria Alfonsa (1985): Individual differences in analogical problem solving, Person. individ. Diff., 18 (5), 611-619.

23. Rittschof, Kent A. (2008): Field dependence-independence as visuospatial and executive functioning in working memory: implications for instructional systems design and research, Education Tech Research Dev. DOI 10.1007/s11423-008-9093-6.

24. Frank, Bernard M. (1984): Effect of field independence-dependence and study technique on learning from a lecture, American Educational Research Journal, 12 (3), 669-678.

25. Hestenes, David (1987) Toward a modelling theory of physics instruction, Am. J. Phys, 55 (5), 440-454

26. Sorby, Sheryl A. (1999) Developing 3-D Spatial Visualization Skills, Engineering Design Graphics Journal, 63 (2), 21-32.

27. Leopold, Cornelie; Gorska, Renata A.; Sorby, Sheryl A. (2001): International Experiences in Developing the Spatial Visualization Abilities of Engineering Students, Journal for Geometry and Graphics, 5 (1), 81-91

28. Towle, Erick; Mann, Jennifer; and Kinsey, Brad (2005) Work In Progress - Development of Tools to Improve the Spatial Ability of Engineering Students, Frontiers in education Conference (FIE 2005), Indianapolis October 19-22.

29. Mohler, James $L$ (2008) The Impact of Visualization Methodology on Spatial Problem Solutions Among High and Low Visual Achievers, Journal of Industrial Technology, 24 (1), January 2008 through March 2008, 1-9. 